Preprint de "On the invariance of the set of stable matchings with respect to substitutable preference profiles," de Ruth Martínez, Jordi Massó, Alejandro Neme i Jorge Oviedo. International Journal of Game Theory 36, 497-518 (2008). Lliurat a Springer el març de 2007. 


\title{
On the Invariance of the Set of Stable Matchings with Respect to Substitutable Preference Profiles*
}

\author{
by \\ Ruth Martínez ${ }^{\dagger}$, Jordi Massó ${ }^{\ddagger}$, Alejandro Neme ${ }^{\dagger}$, and Jorge Oviedo ${ }^{\dagger}$
}

March 6, 2007

\begin{abstract}
For the many-to-one matching model we give a procedure to partition the set of substitutable preference profiles into equivalence classes with the property that all profiles in the same class have the same set of stable matchings. This partition allows to reduce the amount of information required by centralized stable mechanisms.
\end{abstract}

Keywords: Matching, Stability, Substitutable Preferences, Semilattice. Journal of Economic Literature Classification Numbers: C78, D71, D78.

${ }^{*}$ We are very grateful to José Alcalde, Guillaume Haeringer, Flip Klijn, Joana Pais, and two anonymous referees for their helpful comments. The work of R. Martínez, A. Neme, and J. Oviedo is partially supported by the Universidad Nacional de San Luis through grant 319502, by the Consejo Nacional de Investigaciones Científicas y Técnicas CONICET through grant PICT-02114, and by the Agencia Nacional de Promoción Científica y Técnica through grants 03-10814 and PAV-008. The work of J. Massó is partially supported by the Ministerio de Educación y Ciencia (Spain), through grant SEJ2005-01481/ECON and FEDER and project CONSOLIDER-INGENIO 2010 (CDS2006-00016), and by the Generalitat de Catalunya through grant SGR2005-00454 and through the Barcelona Economics Program (XREA). All authors acknowledge financial support from the grants PCI España-Iberoamérica 2005 and 2006 (Programa de Cooperación Interuniversitaria de la Agencia Española de Cooperación Internacional-AECI).

†Instituto de Matemática Aplicada de San Luis. Universidad Nacional de San Luis and CONICET. Ejército de los Andes 950. 5700, San Luis, Argentina. E-mails: martinez@unsl.edu.ar, aneme@unsl.edu.ar, and joviedo@unsl.edu.ar

†DDepartament d’Economia i d’Història Econòmica and CODE. Universitat Autònoma de Barcelona. 08193, Bellaterra (Barcelona), Spain. E-mail: jordi.masso@uab.es 


\section{Introduction}

The purpose of this paper is to give, for each substitutable preference profile of the manyto-one matching model, a systematic procedure to distinguish between those orderings (on pairs of subsets of workers in the preferences of firms) that are irrelevant for the set of stable matchings from those that, if inverted, the set of stable matchings changes.

The many-to-one matching model consists of two disjoint sets: the set of institutions (like firms, colleges, schools, and hospitals) and the set of individuals (workers, students, children, and medical interns). The assignment problem consists of matching each firm to a subset of workers and each worker to at most one firm in such a way that if a worker is matched to a firm, this firm is matched to a subset of workers that contains this worker (workers as well as firms may remain unmatched). The assignment problem is not trivial because agents have preference relations on potential mates. Each worker has a strict preference relation on the set of firms plus the prospect of remaining unmatched, and each firm has a strict preference relation on the family of subsets of workers (which includes the empty set, interpreted as being unmatched). A preference profile is a list of preference relations, one for each agent.

Stability has consistently been used as the solution concept for matching markets. In particular, many entry-level professional labor markets use a centralized stable mechanism which collects agents' preference relations and proposes a stable matching. The National Resident Matching Program, that matches each year around 20,000 hospital positions and medical students for internship in the USA and Canada, is a well-known example of a centralized stable mechanism. ${ }^{1}$ In many countries students are matched to positions in colleges or schools through centralized mechanisms as well. ${ }^{2}$ But also civil servants, sportsmen, researchers, and many others are often matched using centralized mechanisms. ${ }^{3}$

To be stable, a matching has to be individually rational: agents have to be assigned

\footnotetext{
${ }^{1}$ See Roth (1984a), Roth and Peranson (1999), and Roth (2002) for a description and analysis of this market.

${ }^{2}$ See, for instance, Abdulkadiroğlu and Sönmez (2003), Abdulkadiroğlu, Pathak, and Roth (2005), Abdulkadiroğlu, Pathak, Roth, and Sönmez (2005 and 2006).

${ }^{3}$ See Roth and Xing (1994) for a discussion of many centralized matching markets. Romero-Medina and Triossi (2005) perform a strategic analysis of a matching market in Spain in which young researchers are matched to research institutions by means of a partially centralized mechanism.
} 
to acceptable partners; otherwise, if matching is voluntary, the match will not last. In addition, to be stable, a matching has to be pairwise stable: there should not exist any pair formed by a firm and a worker that are not matched to each other, such that the worker would prefer the firm to the current match and the firm would like to add the worker to the set of workers that it is matched to (perhaps after firing some of these workers).

In the marriage model and the college admissions problem the set of stable matchings is always non-empty. ${ }^{4}$ However, for some preference profiles of the general many-to-one matching model the set of stable matchings is empty. All these preference profiles share the feature that some firm considers some workers as complements. Kelso and Crawford (1982) defined the notion of substitutability as the absence of complementarities. Substitutability says that the desirability of a worker in a particular set does not come from the presence of another worker in that set; i.e., the firm still wants to hire the worker even when the other worker is not available anymore. If the preference profile is substitutable (i.e., each firm has a substitutable preference relation) the set of stable matchings is non-empty. Hence, we will assume that preference profiles are substitutable.

The aim of this paper is to understand when the set of stable matchings changes in response to changes in the preference relations of firms. Take a preference profile and a firm. Consider two subsets of workers $S$ and $S^{\prime}$ and assume that the firm prefers $S$ to $S^{\prime}$. Replace in the preference profile the preference relation of the firm with a new preference relation in which the firm prefers $S^{\prime}$ to $S$ and all other orderings remain the same. Depending on the preference profile and the two subsets $S$ and $S^{\prime}$ the set of stable matchings may either change or remain the same. We will give a procedure to identify those orderings between pairs of subsets of workers that, if inverted, the set of stable matchings remains the same for all possible preference relations of the other agents; i.e., orderings between pairs of subsets of workers that from the point of view of stability are irrelevant. Hence, centralized stable mechanisms do not need to use as input this irrelevant information. Specifically, given the substitutable preference relation $P_{f}$ of firm $f$ on all subsets of workers, define a partial order $\succeq_{P_{f}}$ as follows: ${ }^{5}$ given two subsets of workers $S$ and $S^{\prime}$, we declare that $S$ is preferred

\footnotetext{
${ }^{4}$ These two models were introduced and studied in Gale and Shapley's (1962) seminal paper. The marriage model is the one-to-one matching model and the college admissions problem is the many-to-one matching model with quotas and responsive preferences (a meaningful subclass of substitutable preferences).

${ }^{5} \mathrm{~A}$ (strict) preference relation of a firm is a complete, transitive, and antireflexive binary relation on
} 
(according to the partial order $\succeq_{P_{f}}$ ) to $S^{\prime}$ if and only if $S$ is the best subset (according to $P_{f}$ ) among all subsets of $S \cup S^{\prime}$; otherwise, they are not ordered by $\succeq_{P_{f}}$. It turns out that this partial order is a semilattice on a subfamily of subsets of workers. ${ }^{6}$ We refer to it as the semilattice of the choice of the union. We will prove that it is individually rational, ordered, and closed. A semilattice is individually rational if the partially ordered family of subsets of workers is composed of those sets that are preferred to their subsets. A semilattice is ordered if for all subsets $S$ and $S^{\prime}$ in the partially ordered family of subsets of workers, the least upper bound (according to $\succeq_{P_{f}}$ ) of $S$ and $S^{\prime}$ coincides with the least upper bound (according to $\succeq_{P_{f}}$ ) of all subsets of $S \cup S^{\prime}$, and in addition, this set is contained in $S \cup S^{\prime}$. A semilattice is closed if all subsets of each set in the family are themselves elements of the family. Now, suppose we start with an individually rational, ordered, and closed semilattice and we (strongly) extend it to a preference relation by maintaining all orderings of the partial order and declaring a particular order on all unordered pairs. Observe that in general there will be many different strong extensions of a semilattice. However, all strong extensions will be substitutable. Yet, for each strong extension we can obtain its corresponding semilattice of the choice of the union. Then, it turns out that all these semilattices are the same and coincide with the original one from which we obtained the strong extensions.

All these properties are relevant because they will be useful to prove that we can partition the set of substitutable preference profiles by grouping together in equivalence classes all profiles including, for each firm, preference relations that are strong extensions of the same semilattice. Note that, given a preference relation $P_{w}$ of worker $w$, we could similarly construct the partial order $\succeq_{P_{w}}$ on the set of acceptable firms. However, this partial order $\succeq_{P_{w}}$ is trivially a semilattice on the set of acceptable firms because it coincides with the initial complete preference relation $P_{w}$ (on the set of acceptable firms) since the choice of the union of two different firms is always equal to the best of the two firms. Thus, from the point of view of the workers' preference relations all orderings (between pairs of acceptable firms)

the family of all subsets of workers. A partial order of a firm is a reflexive, transitive, and antisymmetric binary relation on a family of subsets of workers. Observe that, in general, a partial order is not complete; i.e., some pair of subsets of workers may not be comparable.

${ }^{6} \mathrm{~A}$ semilattice is a partially ordered set with the property that the least upper bound of any pair of elements in the set exists. 
are relevant for the set of stable matchings. This is the reason why preference relations of workers will remain fixed while identifying equivalence classes of preferences of firms. In particular, Theorem 1 says that the set of stable matchings is invariant across substitutable preference profiles that belong to the same equivalence class. Hence, from the point of view of stability information contained in the complete preference relations of firms is irrelevant since only those orderings kept by the profile of semilattices of the choice of the union matter. Thus, centralized stable mechanisms could disregard this irrelevant information.

The paper is organized as follows. In Section 2 we present the preliminary notation and definitions. In Section 3 we describe our results by means of an example, define the notion of a semilattice, and state its main properties. In Section 4 we define the notion of a strong extension of a semilattice and present some preliminary results. In Section 5 we state and prove the main result of the paper: Theorem 1, an invariance result, under substitutable preference profiles, for the set of stable matchings. Finally, in Section 6 we conclude with a general description of the procedure that partitions the set of substitutable preference profiles.

\section{Preliminaries}

\subsection{Agents, Preference Relations, and Matchings}

There are two disjoint sets of agents, a set of $n$ firms $F=\left\{f_{1}, \ldots, f_{n}\right\}$ and a set of $m$ workers $W=\left\{w_{1}, \ldots, w_{m}\right\}$. Generic elements of both sets are denoted, respectively, by $f$ and by $w$. A generic agent will be denoted by $v \in V \equiv F \cup W$. Firms will hire sets of workers (possibly empty) and workers will work for at most one firm. Thus, each worker $w \in W$ has a strict, transitive, and complete preference relation $P_{w}$ over $F \cup\{\emptyset\}$, and each firm $f \in F$ has a strict, transitive, and complete preference relation $P_{f}$ over $2^{W}$. Preference profiles are $(n+m)$-tuples of preference relations and they are represented by $P=\left(P_{f_{1}}, \ldots, P_{f_{n}} ; P_{w_{1}}, \ldots, P_{w_{m}}\right)$. Given a preference profile $P$ and $f$ 's preference relation $P_{f}^{\prime}$, we will denote by $\left(P_{f}^{\prime}, P_{-f}\right)$ the original preference profile $P$ after replacing $P_{f}$ by $P_{f}^{\prime}$. Given a preference relation of a firm $P_{f}$, the subsets of workers preferred to the empty set by $f$ are called acceptable. Similarly, given a preference relation of a worker $P_{w}$, the firms preferred by $w$ to the empty set are called acceptable. By convention, we declare the empty 
set as being acceptable for all agents.

A market is a triple $(F, W, P)$. The assignment problem consists of matching workers with firms, keeping the bilateral nature of their relationship and allowing for the possibility that both firms and workers may remain unmatched. Formally,

Definition 1 A matching $\mu$ is a mapping from the set $F \cup W$ into the set of all subsets of $F \cup W$ such that for all $w \in W$ and all $f \in F$ :

1. Either $|\mu(w)|=1$ and $\mu(w) \cap F \neq \emptyset$ or else $\mu(w)=\emptyset$.

2. $\mu(f) \in 2^{W}$.

3. $\mu(w)=\{f\}$ if and only if $w \in \mu(f) .^{7}$

Let $\mathcal{M}$ be the set of matchings. We say that agent $v \in F \cup W$ is unmatched at matching $\mu$ if $\mu(v)=\emptyset$. Otherwise, $v$ is matched at $\mu$. Given a matching $\mu$ and a subset of agents $C \subseteq V$, define $\mu(C)=\{v \in V \mid \mu(v) \cap C \neq \emptyset\}$.

Let $P_{f}$ be a preference relation of firm $f$. Given a set of workers $S \subseteq W$, let $C h\left(S, P_{f}\right)$ denote firm $f$ 's most-preferred subset of $S$ according to its preference relation $P_{f}$. Generically we refer to this set as the choice set.

\subsection{Stability and Substitutable Preferences}

Let $(F, W, P)$ be a market. A matching $\mu$ is blocked by worker $w$ if $\emptyset P_{w} \mu(w)$. A matching $\mu$ is blocked by firm $f$ if $\mu(f) \neq C h\left(\mu(f), P_{f}\right)$. A matching is individually rational if it is not blocked by any individual agent. We will denote the set of individually rational matchings by $\operatorname{IR}(P)$. A firm-worker pair $(f, w)$ is a pairwise block of matching $\mu$ if $w \notin \mu(f)$, $f P_{w} \mu(w)$, and $w \in C h\left(\mu(f) \cup\{w\}, P_{f}\right)$.

Definition 2 A matching $\mu$ is stable if it is not blocked by any individual agent nor any firm-worker pair.

Given a preference profile $P$, we denote the set of stable matchings by $S(P)$. There are preference profiles with the property that the set of stable matchings is empty. Following the literature we will assume that firms have substitutable preference relations.

\footnotetext{
${ }^{7}$ With a slight abuse of notation, we often use $\mu(w)$ as an element of $F$, and write $\mu(w)=f$ instead of $\mu(w)=\{f\}$.
} 
Definition 3 A firm $f$ 's preference relation $P_{f}$ satisfies substitutability if for any set $S$ containing workers $w$ and $w^{\prime}\left(w \neq w^{\prime}\right)$, if $w \in C h\left(S, P_{f}\right)$ then $w \in C h\left(S \backslash\left\{w^{\prime}\right\}, P_{f}\right)$.

A preference profile $P$ is substitutable if for each firm $f$, the preference relation $P_{f}$ satisfies substitutability. For any substitutable preference profile $P, S(P) \neq \emptyset$ and, for all $\mu \in S(P), \mu_{F} R_{f} \mu R_{f} \mu_{W}$ for all $f \in F$ and $\mu_{W} R_{w} \mu R_{w} \mu_{F}$ for all $w \in W .8$

The deferred-acceptance algorithm defined by Gale and Shapley (1962) produces, for each substitutable preference profile $P$, either $\mu_{F}$ or $\mu_{W}$ depending on the side of the market that makes the offers. At any step of the algorithm in which firms make offers, each firm $f$ proposes to the choice set of the set of workers that have not already rejected $f$ during previous steps, while a worker $w$ accepts the most-preferred firm among the set of current offers plus the firm provisionally matched to $w$ in the previous step (if any). The algorithm stops at the step when either all offers are accepted or firms have no more acceptable subsets of workers to whom they want to make an offer; the provisional matching becomes then definite and is the stable matching $\mu_{F}$. Similarly, if workers make offers, the outcome of the algorithm is the stable matching $\mu_{W}$.

\section{Invariance of the Set of Stable Matchings and Semi- lattices}

\subsection{An Example}

Our goal is to identify conditions on substitutable preference profiles under which the set of stable matchings is invariant. Specifically, we aim to give a simple procedure to partition the set of substitutable preference profiles into equivalence classes with the property that all profiles in the same class have the same set of stable matchings. Before proceeding, we present an example that illustrates the main ideas of this procedure.

\footnotetext{
${ }^{8}$ See Kelso and Crawford (1982) and Roth (1984b). The matchings $\mu_{F}$ and $\mu_{W}$ are called, respectively, the firms-optimal stable matching and the workers-optimal stable matching. We are following the convention of extending preferences from the original sets $\left(2^{W}\right.$ and $\left.F \cup\{\emptyset\}\right)$ to the set of matchings. However, we now have to consider weak preference relations since matchings $\mu$ and $\mu^{\prime}$ may associate to an agent the same partner. This weak preference relation of agent $v$ is denoted by $R_{v}$.
} 
Example 1 Let $F=\left\{f_{1}, f_{2}\right\}$ be the set of firms and $W=\left\{w_{1}, w_{2}, w_{3}\right\}$ be the set of workers. Consider the substitutable preference profile $P=\left(P_{f_{1}}, P_{f_{2}} ; P_{w_{1}}, P_{w_{2}}, P_{w_{3}}\right)$,

$\begin{array}{ccccc}\frac{P_{f_{1}}}{\left\{w_{3}\right\}} & \left\{{P_{f_{2}}}_{1}, w_{2}\right\} & \frac{P_{w_{1}}}{f_{1}} & \frac{P_{w_{2}}}{f_{1}} & \frac{P_{w_{3}}}{f_{2}} \\ \left\{w_{1}, w_{2}\right\} & \left\{w_{1}, w_{3}\right\} & f_{2} & f_{2} & f_{1} \\ \left\{w_{1}\right\} & \left\{w_{2}, w_{3}\right\} & \emptyset & \emptyset & \emptyset \\ \left\{w_{2}\right\} & \left\{w_{3}\right\} & & & \\ \emptyset & \left\{w_{1}\right\} & & & \\ & \left\{w_{2}\right\} & & & \\ \emptyset & & & \end{array}$

where we only list acceptable partners in decreasing order (all missing subsets of workers in the corresponding preference relations of firms are not acceptable and, by individual rationality of stable matchings, their relative orderings are irrelevant from the point of view of stability). It is easy to check that $S(P)=\left\{\mu_{1}, \mu_{2}\right\}$, where $\mu_{1}\left(f_{1}\right)=\left\{w_{1}, w_{2}\right\}$, $\mu_{1}\left(f_{2}\right)=\left\{w_{3}\right\}, \mu_{2}\left(f_{1}\right)=\left\{w_{3}\right\}$, and $\mu_{2}\left(f_{2}\right)=\left\{w_{1}, w_{2}\right\}$. Consider now the two profiles $P^{\prime}$ and $P^{\prime \prime}$ in which only $f_{2}$ 's preference relation $P_{f_{2}}$ has changed to $P_{f_{2}}^{\prime}$ and to $P_{f_{2}}^{\prime \prime}$, respectively; that is, $P^{\prime}=\left(P_{f_{2}}^{\prime}, P_{-f_{2}}\right)$ and $P^{\prime \prime}=\left(P_{f_{2}}^{\prime \prime}, P_{-f_{2}}\right)$, where

$\begin{array}{cc}\frac{P_{f_{2}}^{\prime}}{\left\{w_{1}, w_{2}\right\}} & \left\{\frac{P_{f_{2}}^{\prime \prime}}{\left.w_{1}, w_{3}\right\}}\right. \\ \left\{w_{2}, w_{3}\right\} & \left\{w_{1}, w_{2}\right\} \\ \left\{w_{1}, w_{3}\right\} & \left\{w_{2}, w_{3}\right\} \\ \left\{w_{3}\right\} & \left\{w_{3}\right\} \\ \left\{w_{1}\right\} & \left\{w_{1}\right\} \\ \left\{w_{2}\right\} & \left\{w_{2}\right\} \\ \emptyset & \emptyset .\end{array}$

Note that $P_{f_{2}}$ and $P_{f_{2}}^{\prime}$ differ only on the ordering of the sets $\left\{w_{1}, w_{3}\right\}$ and $\left\{w_{2}, w_{3}\right\}$ while $P_{f_{2}}$ and $P_{f_{2}}^{\prime \prime}$ differ only on the ordering of the sets $\left\{w_{1}, w_{2}\right\}$ and $\left\{w_{1}, w_{3}\right\}$. However, the replacement of $P_{f_{2}}$ by $P_{f_{2}}^{\prime}$ does not produce any effect on the set of stable matchings since $S\left(P^{\prime}\right)=S(P)=\left\{\mu_{1}, \mu_{2}\right\}$, while the replacement of $P_{f_{2}}$ by $P_{f_{2}}^{\prime \prime}$ changes the set of stable 
matchings since $S\left(P^{\prime \prime}\right)=\left\{\mu_{1}\right\}$ (observe that $\mu_{2} \notin S\left(P^{\prime \prime}\right)$ because $\left(f_{2}, w_{3}\right)$ is a pairwise block of $\mu_{2}$ ).

We will have to differentiate between irrelevant versus relevant orderings (i.e., comparisons between two sets of workers) in $P_{f}$. The ordering $S P_{f} S^{\prime}$ is irrelevant for stability if for each $P_{f}^{\prime}$ that agrees with $P_{f}$ except on the ordering of $S$ and $S^{\prime}, S\left(P_{f}^{\prime}, P_{-f}\right)=S\left(P_{f}, P_{-f}\right)$ for all $P_{-f}$; otherwise, the ordering $S P_{f} S^{\prime}$ is relevant for stability. For this purpose, we will have to consider a partial order $\succeq_{P_{f}}$, which will leave as unordered those pairs that are irrelevantly ordered by $P_{f}$, keeping all relevant orderings in $P_{f} .{ }^{9}$ Second, to understand which orderings are irrelevant and which ones are relevant we will have to look at very special properties of the partial order $\succeq_{P_{f}}$. For instance, we associate with the preference relation $P_{f_{2}}$ a partial order $\succeq_{P_{f_{2}}}$ on the subfamily of subsets $A_{P_{f_{2}}}=\left\{S \in 2^{W} \mid S=C h\left(S, P_{f_{2}}\right)\right\}$ (i.e., $A_{P_{f_{2}}}=2^{W} \backslash\left\{w_{1}, w_{2}, w_{3}\right\}$ ) as follows: ${ }^{10}$ for all $S, S^{\prime} \in A_{P_{f_{2}}}$,

$$
S \succeq_{P_{f_{2}}} S^{\prime} \text { if and only if } S=C h\left(S \cup S^{\prime}, P_{f_{2}}\right)
$$

Observe that $A_{P_{f_{2}}}=A_{P_{f_{2}}^{\prime}}$ and for all $S, S^{\prime} \in A_{P_{f_{2}}}, S \succeq_{P_{f_{2}}} S^{\prime}$ if and only if $S \succeq_{P_{f_{2}}^{\prime}} S^{\prime}$; namely, $\succeq_{P_{f_{2}}}=\succeq_{P_{f_{2}}^{\prime}}$, because

\footnotetext{
${ }^{9}$ Alkan (2001) also considers partial orders instead of complete preference relations to study the lattice structure of the set of stable matchings of a many-to-one matching model.

${ }^{10}$ Blair (1988) uses a similar construction to establish the lattice structure of the set of stable matchings in the many-to-many model introduced and studied by Roth (1984b and 1985). Fleiner (2003) uses a partial order to study the set of stable matchings as fixed points. Echenique and Oviedo (2006) also uses this partial order to identify a condition (strong substitutability) on preference relations that guarantee the non-emptyness of the set of set-wise stable matchings in the many-to-many matching model.
} 


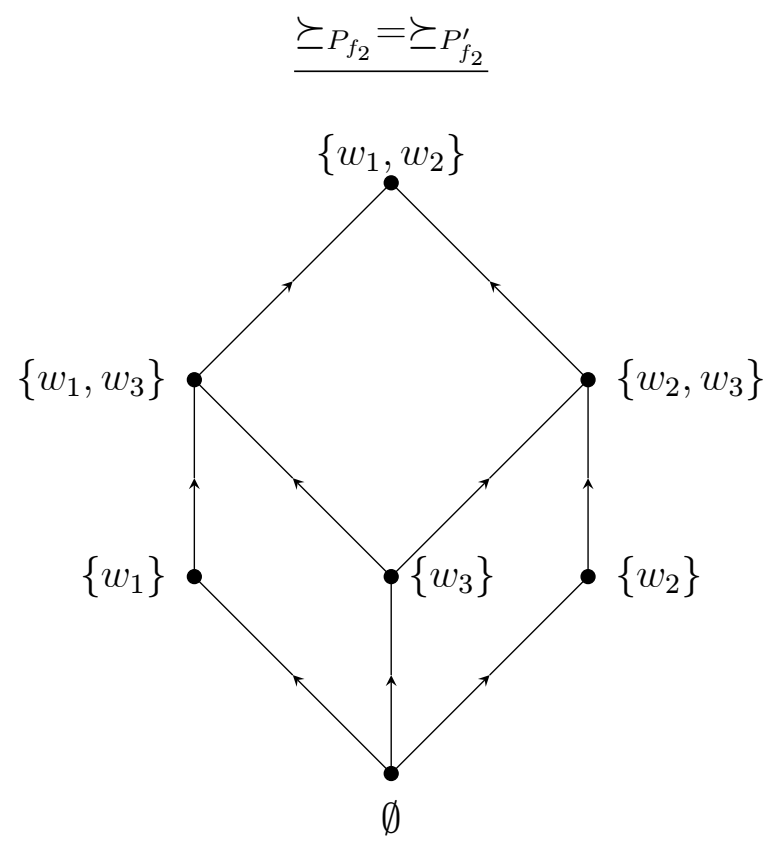

where $S \rightarrow S^{\prime}$ means that $S^{\prime} \succ S\left(S^{\prime} \succeq S\right.$ and $\left.S \neq S^{\prime}\right)$ and omitted nonempty subsets are unordered with respect to all other subsets of $A_{P_{f_{2}}}$. However,

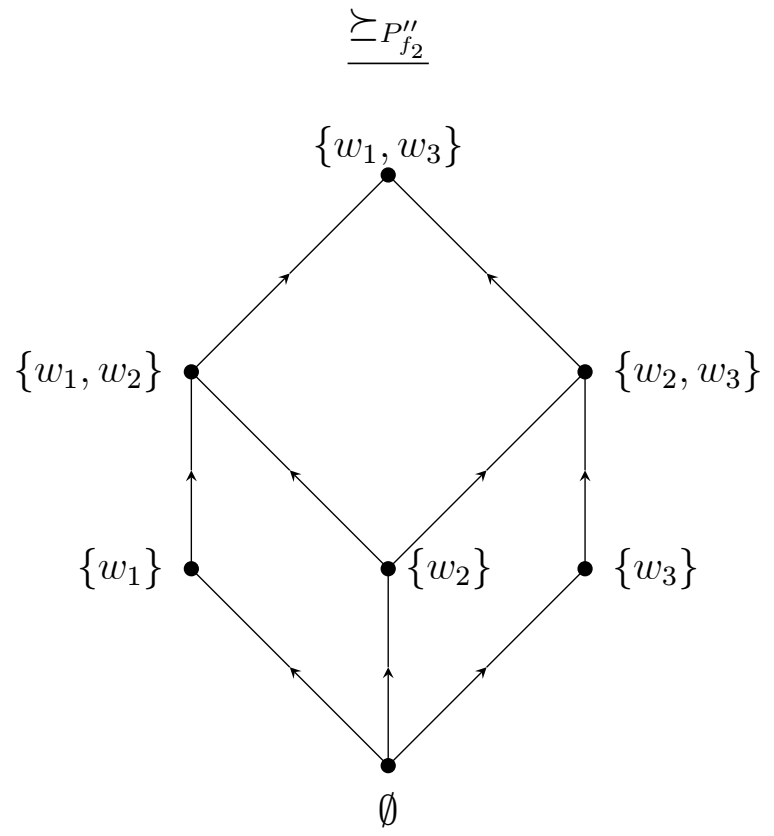

Observe that, since $\left\{w_{1}, w_{3}\right\} \succeq_{P_{f_{2}}^{\prime \prime}}\left\{w_{1}, w_{2}\right\}$ but $\left\{w_{1}, w_{2}\right\} \succeq_{P_{f_{2}}}\left\{w_{1}, w_{3}\right\}, \succeq_{P_{f_{2}}^{\prime \prime}} \neq \succeq_{P_{f_{2}}}$. Con- 
sider

$$
\begin{gathered}
\frac{P_{f_{2}}^{\prime \prime \prime}}{\left\{w_{1}, w_{2}\right\}} \\
\left\{w_{1}, w_{3}\right\} \\
\left\{w_{2}, w_{3}\right\} \\
\left\{w_{2}\right\} \\
\left\{w_{1}\right\} \\
\left\{w_{3}\right\}
\end{gathered}
$$

$\emptyset$

which differs from $P_{f_{2}}, P_{f_{2}}^{\prime}$, and $P_{f_{2}}^{\prime \prime}$. Since $\succeq_{P_{f_{2}}^{\prime \prime \prime}}=\succeq_{P_{f_{2}}^{\prime}}=\succeq_{P_{f_{2}}} \neq \succeq_{P_{f_{2}}^{\prime \prime}}$ we will be able to deduce, as a consequence of Theorem 1 , that $S\left(P_{f_{2}}^{\prime \prime \prime}, \hat{P}_{-f_{2}}\right)=S\left(P_{f_{2}}^{\prime}, \hat{P}_{-f_{2}}\right)=S\left(P_{f_{2}}, \hat{P}_{-f_{2}}\right)$ for all $\hat{P}_{-f_{2}}$, and that there exists $\bar{P}_{-f_{2}}$ such that $S\left(P_{f_{2}}^{\prime \prime}, \bar{P}_{-f_{2}}\right) \neq S\left(P_{f_{2}}, \bar{P}_{-f_{2}}\right)$.

Subsection 3.2 below presents formally the notion of a semilattice which will be needed to state our results. We adapt this notion to our setting where the partially ordered set is a (finite) subfamily of subsets of workers. ${ }^{11}$

\subsection{Partial Orders, Joins, and Semilattices}

Let $A$ be a non-empty subfamily of subsets of $W$ containing the empty set; i.e., $A \subseteq 2^{W}$ and $\emptyset \in A$. A partial order $\succeq$ on $A$ is a reflexive, transitive, and antisymmetric binary relation on $A$; that is, for all $S, S^{\prime}, S^{\prime \prime} \in A, S \succeq S,\left[S \succeq S^{\prime} \succeq S^{\prime \prime}\right] \Longrightarrow\left[S \succeq S^{\prime \prime}\right]$, and $\left[S \succeq S^{\prime}\right.$ and $\left.S^{\prime} \succeq S\right] \Longrightarrow\left[S=S^{\prime}\right]$. Given $S, S^{\prime} \in A$ we write $S \succ S^{\prime}$ to denote that $S \succeq S^{\prime}$ and $S \neq S^{\prime}$. Then, $\succ$ is a transitive and antireflexive $(S \succ S$ for no $S)$ binary relation on $A$. The set $S$ is called acceptable (according to $\succeq$ ) if $S \succeq \emptyset$. Given a partial order $\succeq$ on $A$ and a subfamily $X \subseteq A$, define the set of upper bounds of $X$ as $u b_{\succeq} X=\left\{S \in A \mid S \succeq S^{\prime}\right.$ for all $\left.S^{\prime} \in X\right\}$ and the least upper bound of $X$ as $l u b_{\succeq} X=T$, where $T \in u b_{\succeq} X$ and, for all $T^{\prime} \in u b_{\succeq} X, T^{\prime} \succeq T$. Given a partial order $\succeq$ on $A$, define the binary operation $\vee$ on $A$ as follows: for $S, S^{\prime} \in A, S \vee S^{\prime}=l u b_{\succeq}\left\{S, S^{\prime}\right\}$. Observe that, in general, lub $\left.l S, S^{\prime}\right\}$ may not exist; for instance, consider $W=\left\{w_{1}, w_{2}, w_{3}\right\}$ and let $A=\left\{\left\{w_{1}\right\},\left\{w_{2}\right\},\left\{w_{3}\right\}, \emptyset\right\}$ and $\succeq$ be such that $\left\{w_{1}\right\} \succ\left\{w_{3}\right\}$ and $\left\{w_{2}\right\} \succ\left\{w_{3}\right\}$. Then, $l u b_{\succeq}\left\{\left\{w_{1}\right\},\left\{w_{2}\right\}\right\}$ does not exist because $u b_{\succeq}\left\{\left\{w_{1}\right\},\left\{w_{2}\right\}\right\}=\emptyset$. However, by the antisymmetry of $\succeq$, if it exists, the $l u b_{\succeq}$ is unique.

\footnotetext{
${ }^{11}$ See Birkhoff (1979) for a general definition.
} 
Definition 4 The triple $L=(A, \succeq, \vee)$ is a semilattice if, for all $S, S^{\prime} \in A, l u b_{\succeq}\left\{S, S^{\prime}\right\}$ exists. $^{12}$

Observe that although the binary operation $\vee$ follows from the partial order $\succeq$, it will be useful to refer to both in the notation of the semilattice. This is because there is an (equivalent) algebraic approach where, instead of starting from the partial order $\succeq$, one can start from a binary operation as follows. A join $\vee$ on $A$ is an idempotent, commutative, and associative binary relation on $A$; that is, for all $S, S^{\prime}, S^{\prime \prime} \in A, S \vee S=S, S \vee S^{\prime}=S^{\prime} \vee S$, and $S \vee\left(S^{\prime} \vee S^{\prime \prime}\right)=\left(S \vee S^{\prime}\right) \vee S^{\prime \prime}$. Given a join $\vee$ on $A$, define the partial order $\succeq$ on $A$ as follows: for all $S, S^{\prime} \in A$,

$$
S \succeq S^{\prime} \text { if and only if } S=S \vee S^{\prime}
$$

Indeed, both approaches are equivalent in the sense that the partial order obtained from $\vee$ is $\succeq$ (i.e., the partial order from which $\vee$ is defined).

\subsection{The Semilattice of the Choice of the Union: Definition and Properties}

Let $f \in F$ and $P_{f}$ be given. Assume $P_{f}$ is substitutable. Define the family of subsets of workers

$$
A_{P_{f}}=\left\{S \in 2^{W} \mid S=C h\left(S, P_{f}\right)\right\}
$$

and the partial order $\succeq_{P_{f}}$ on $A_{P_{f}}$ as follows: for all $S, S^{\prime} \in A_{P_{f}}$,

$$
S \succeq_{P_{f}} S^{\prime} \text { if and only if } S=C h\left(S \cup S^{\prime}, P_{f}\right) \text {. }
$$

It is easy to see that for any preference relation $P_{f}$ the binary relation $\succeq_{P_{f}}$ is a partial order on $A_{P_{f}}$; i.e., $\succeq_{P_{f}}$ is reflexive, transitive, and antisymmetric. Moreover, Proposition 1 below

\footnotetext{
${ }^{12}$ Specifically, we should have referred to $L=(A, \succeq, \vee)$ as a join-semilattice. Moreover, if a joinsemilattice $L=(A, \succeq, \vee)$ has the property (as it will be in the sequel) that all sets in $A$ are acceptable, then $\emptyset \in A$ is the smallest element of $\succeq$. Birkhoff (page 23, 1979) shows that any join-semilattice with a smallest element is also a lattice. Hence, $L=(A, \succeq, \vee)$ is indeed a lattice (i.e., a join-semilattice with the property that every pair of sets has a geatest lower bound). Since for our pourpose the interesting binary operation is the join $\vee$, not the meet $\wedge$, we will not emphasize this fact and still refer to $L=(A, \succeq, \vee)$ as a semilattice.
} 
says that the triple $\left(A_{P_{f}}, \succeq_{P_{f}}, \vee_{P_{f}}\right)$ is a semilattice. We call it the semilattice of the choice of the union.

Proposition 1 Let $P_{f}$ be a substitutable preference relation. Then, the triple $\left(A_{P_{f}}, \succeq_{P_{f}}\right.$ ,$\vee_{P_{f}}$ ) is a semilattice.

Proof We will show that for all $S, S^{\prime} \in A_{P_{f}}, l u b_{\succeq_{P_{f}}}\left\{S, S^{\prime}\right\}$ exists by showing that

$$
l u b_{\succeq_{P_{f}}}\left\{S, S^{\prime}\right\}=C h\left(S \cup S^{\prime}, P_{f}\right) .
$$

Let $X=S \cup S^{\prime}$ and $Y=C h\left(S \cup S^{\prime}, P_{f}\right) \cup S$. Clearly, $C h\left(X, P_{f}\right) \subseteq Y \subseteq X$. Hence, by condition (2.6) in Blair (1988), ${ }^{13} C h\left(X, P_{f}\right)=C h\left(Y, P_{f}\right)$. By definition of $\succeq_{P_{f}}, C h(S \cup$ $\left.S^{\prime}, P_{f}\right) \succeq_{P_{f}} S$. Similarly, $C h\left(S \cup S^{\prime}, P_{f}\right) \succeq_{P_{f}} S^{\prime}$. Thus, $C h\left(S \cup S^{\prime}, P_{f}\right)$ is an upper bound of $\left\{S, S^{\prime}\right\}$. Let $T$ be an upper bound of $\left\{S, S^{\prime}\right\}$ and assume,

$$
C h\left(S \cup S^{\prime}, P_{f}\right) \succeq_{P_{f}} T
$$

Since $T$ is an upper bound of $\left\{S, S^{\prime}\right\}, T \succeq_{P_{f}} S$ and $T \succeq_{P_{f}} S^{\prime}$. By the definition of $\succeq_{P_{f}}$,

$$
T=C h\left(T \cup S, P_{f}\right)=C h\left(T \cup S^{\prime}, P_{f}\right) .
$$

Hence, from (5),

$$
\begin{aligned}
C h\left(S \cup S^{\prime}, P_{f}\right) & =C h\left(C h\left(S \cup S^{\prime}, P_{f}\right) \cup T, P_{f}\right) & & \text { by the definition of } \succeq_{P_{f}} \\
& =C h\left(S \cup S^{\prime} \cup T, P_{f}\right) & & \text { by Proposition } 2.3 \text { in Blair }(1988)^{14} \\
& =C h\left(S \cup C h\left(S^{\prime} \cup T, P_{f}\right), P_{f}\right) & & \text { by Proposition } 2.3 \text { in Blair }(1988) \\
& =C h\left(S \cup T, P_{f}\right) & & \text { by }(6) \\
& =T & & \text { by }(6) .
\end{aligned}
$$

Given $P_{f}$, we denote by $L_{P_{f}}$ the semilattice of the choice of the union $\left(A_{P_{f}}, \succeq_{P_{f}}, \vee_{P_{f}}\right)$ obtained by conditions (2) and (3). In Example 2 below we show that the conclusion of Proposition 1 does not hold for non-substitutable preference relations.

\footnotetext{
${ }^{13}$ Condition (2.6) in Blair (1988) says that for all $P_{f}$ and all $X, Y \in 2^{W}, C h\left(X, P_{f}\right) \subseteq Y \subseteq X$ implies $C h\left(X, P_{f}\right)=C h\left(Y, P_{f}\right)$.

${ }^{14}$ Proposition 2.3 in Blair (1988) says that the choice set of any substitutable preference relation $P_{f}$ has the property that for all $X, Y \in 2^{W}, C h\left(X \cup Y, P_{f}\right)=C h\left(C h\left(X, P_{f}\right) \cup Y, P_{f}\right)$.
} 
Example 2 Let $W=\left\{w_{1}, w_{2}, w_{3}, w_{4}\right\}$ be the set of workers. Consider the preference relation

$$
\begin{gathered}
\frac{P_{f}}{\left\{w_{1}, w_{2}\right\}} \\
\left\{w_{3}, w_{4}\right\} \\
\left\{w_{4}\right\} \\
\left\{w_{3}\right\} \\
\left\{w_{1}\right\} \\
\left\{w_{2}\right\}
\end{gathered}
$$

$\emptyset$.

Observe that $P_{f}$ is not substitutable because $w_{1} \in C h\left(\left\{w_{1}, w_{2}, w_{3}, w_{4}\right\}, P_{f}\right)$ and $w_{1} \notin$ $C h\left(\left\{w_{1}, w_{3}, w_{4}\right\}, P_{f}\right)$. Note that $A_{P_{f}}=\left\{\left\{w_{1}, w_{2}\right\},\left\{w_{3}, w_{4}\right\},\left\{w_{1}\right\},\left\{w_{2}\right\},\left\{w_{3}\right\},\left\{w_{4}\right\}, \emptyset\right\}$. Since $C h\left(\left\{w_{1}\right\} \cup\left\{w_{2}\right\}, P_{f}\right)=\left\{w_{1}, w_{2}\right\}, C h\left(\left\{w_{1}\right\} \cup\left\{w_{2}\right\}, P_{f}\right)$ is neither $\left\{w_{1}\right\}$ nor $\left\{w_{2}\right\}$ and since $C h\left(\left\{w_{3}\right\} \cup\left\{w_{4}\right\}, P_{f}\right)=\left\{w_{3}, w_{4}\right\}, C h\left(\left\{w_{3}\right\} \cup\left\{w_{4}\right\}, P_{f}\right)$ is neither $\left\{w_{3}\right\}$ nor $\left\{w_{4}\right\}$. Thus, $\left\{w_{1}\right\} \nsucceq_{P_{f}}\left\{w_{2}\right\},\left\{w_{2}\right\} \nsucceq_{P_{f}}\left\{w_{1}\right\},\left\{w_{3}\right\} \nsucceq_{P_{f}}\left\{w_{4}\right\}$, and $\left\{w_{4}\right\} \nsucceq_{P_{f}}\left\{w_{3}\right\}$. On the other hand, $C h\left(\left\{w_{1}\right\} \cup\left\{w_{i}\right\}, P_{f}\right)=C h\left(\left\{w_{2}\right\} \cup\left\{w_{i}\right\}, P_{f}\right)=\left\{w_{i}\right\}$, for $i=3,4$, imply $\left\{w_{4}\right\} \succeq_{P_{f}}\left\{w_{1}\right\},\left\{w_{4}\right\} \succeq_{P_{f}}\left\{w_{2}\right\},\left\{w_{3}\right\} \succeq_{P_{f}}\left\{w_{1}\right\}$, and $\left\{w_{3}\right\} \succeq_{P_{f}}\left\{w_{2}\right\}$. Thus, $\left\{w_{3}\right\},\left\{w_{4}\right\} \in u b_{\succeq_{P_{f}}}\left\{\left\{w_{1}\right\},\left\{w_{2}\right\}\right\}$. Since $\left\{w_{3}\right\}$ and $\left\{w_{4}\right\}$ are not ordered through $\succeq_{P_{f}}$ and $\left\{w_{1}, w_{2}\right\} \succeq_{P_{f}}\left\{w_{i}\right\}$ and $\left\{w_{3}, w_{4}\right\} \succeq_{P_{f}}\left\{w_{i}\right\}$, for $i=3,4, l u b_{\succeq_{P_{f}}}\left\{\left\{w_{1}\right\},\left\{w_{2}\right\}\right\}$ does not exist. Hence, the partial order $\succeq_{P_{f}}$ on $A_{P_{f}}$ is not a semilattice.

Since the partial order $\succeq_{P_{f}}$ inherits rationality properties of the choice sets, it may also satisfy some additional properties.

First, to define the notion of stability of a matching $\mu$ we have assumed that a firm $f$ has the possibility, when confronted with the set $\mu(f)$, of choosing the best subset of workers in $\mu(f)$ according to $P_{f}$. Hence, we would like that the semilattice $L_{P_{f}}$ has the following property.

Definition 5 A semilattice $L=(A, \succeq, \vee)$ is individually rational if for all $S, S^{\prime} \in A$ such that $S^{\prime} \subseteq S, S \succeq S^{\prime}$

Note that if a semilattice $L=(A, \succeq, \vee)$ is individually rational then $A$ only contains acceptable subsets of workers. 
Second, given a semilattice $L=(A, \succeq, \vee)$ we want that, for any two subsets $S, S^{\prime} \in A$, the least upper bound of $\left\{S, S^{\prime}\right\}$ coincides with the least upper bound of the family of subsets of $S \cup S^{\prime}$ in $A$ and, in turn, it is a subset of $S \cup S^{\prime}$.

Definition 6 A semilattice $L=(A, \succeq, \vee)$ is ordered if for all $S, S^{\prime} \in A, l u b_{\succeq}\left\{S, S^{\prime}\right\}=$ $l u b_{\succeq}\left\{T \in A \mid T \subseteq S \cup S^{\prime}\right\} \subseteq S \cup S^{\prime}$.

In Example 3 below we illustrate the two notions.

Example 3 Let $W=\left\{w_{1}, w_{2}, w_{3}\right\}$ be the set of workers and assume that the subfamily of subsets is $A=\left\{\left\{w_{1}, w_{2}\right\},\left\{w_{2}, w_{3}\right\},\left\{w_{1}, w_{3}\right\},\left\{w_{1}, w_{2}, w_{3}\right\}, \emptyset\right\} \subset 2^{W}$. Consider the following partial order $\succeq$ on $A$

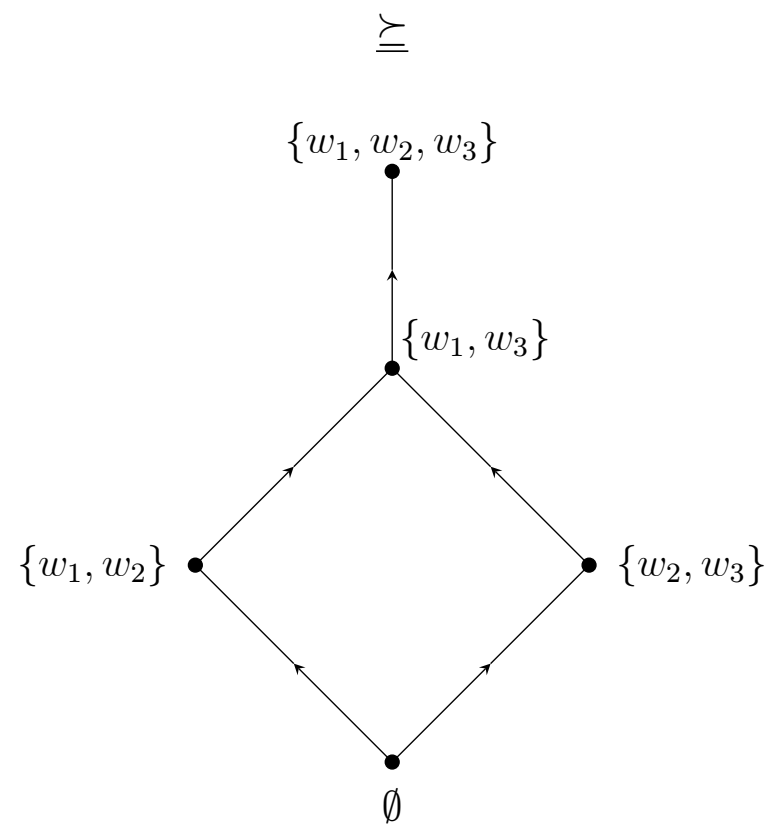

The semilattice $L=(A, \succeq, \vee)$ is individually rational but it is not ordered since $l u b_{\succeq}\left\{\left\{w_{1}, w_{2}\right\},\left\{w_{1}, w_{3}\right\}\right\}=\left\{w_{1}, w_{3}\right\} \neq\left\{w_{1}, w_{2}, w_{3}\right\}=l u b_{\succeq}\left\{T \in A \mid T \subseteq\left\{w_{1}, w_{2}\right\} \cup\right.$ $\left.\left\{w_{1}, w_{3}\right\}\right\}$. Consider now the partial order $\succeq^{\prime}$ on $A$ 


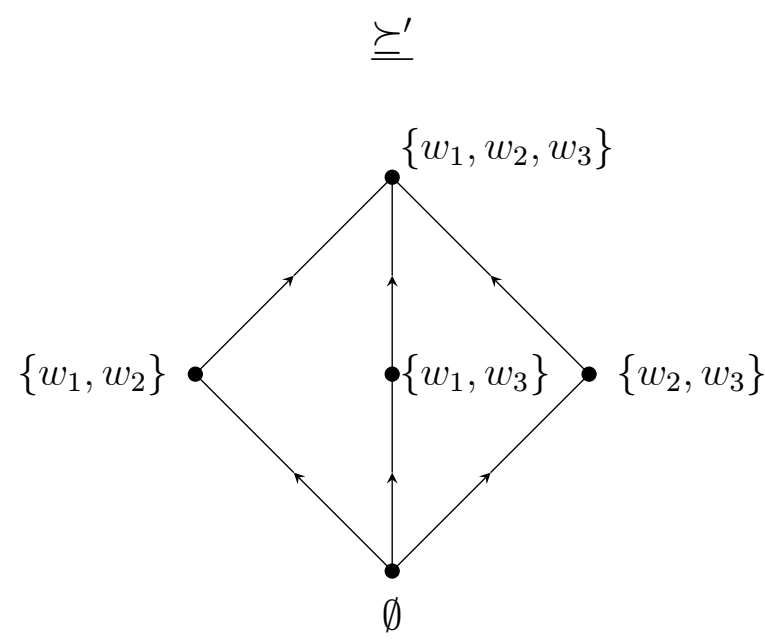

The semilattice $L^{\prime}=\left(A, \succeq^{\prime}, \vee^{\prime}\right)$ is individually rational and ordered since $l u b_{\succeq^{\prime}}\left\{\left\{w_{1}, w_{2}\right\},\left\{w_{1}, w_{3}\right\}\right\}=l u b_{\succeq}\left\{T \in A \mid T \subseteq\left\{w_{1}, w_{2}\right\} \cup\left\{w_{1}, w_{3}\right\}\right\}=\left\{w_{1}, w_{2}, w_{3}\right\}$.

Finally, we will need the notion of a closed semilattice.

Definition 7 A semilattice $L=(A, \succeq, \vee)$ is closed if $S \in A$ and $S^{\prime} \subseteq S$ imply $S^{\prime} \in A$.

Observe that neither $L=(A, \succeq, \vee)$ nor $L^{\prime}=\left(A, \succeq^{\prime}, \vee^{\prime}\right)$ in Example 3 are closed. However, the semilattice $L^{\prime \prime}=\left(A^{\prime \prime}, \succeq^{\prime \prime}, \vee^{\prime \prime}\right)$, where $A^{\prime \prime}=2^{\left\{w_{1}, w_{2}, w_{3}\right\}}$ and $\succeq^{\prime \prime}$ is the following partial order on $A^{\prime \prime}$

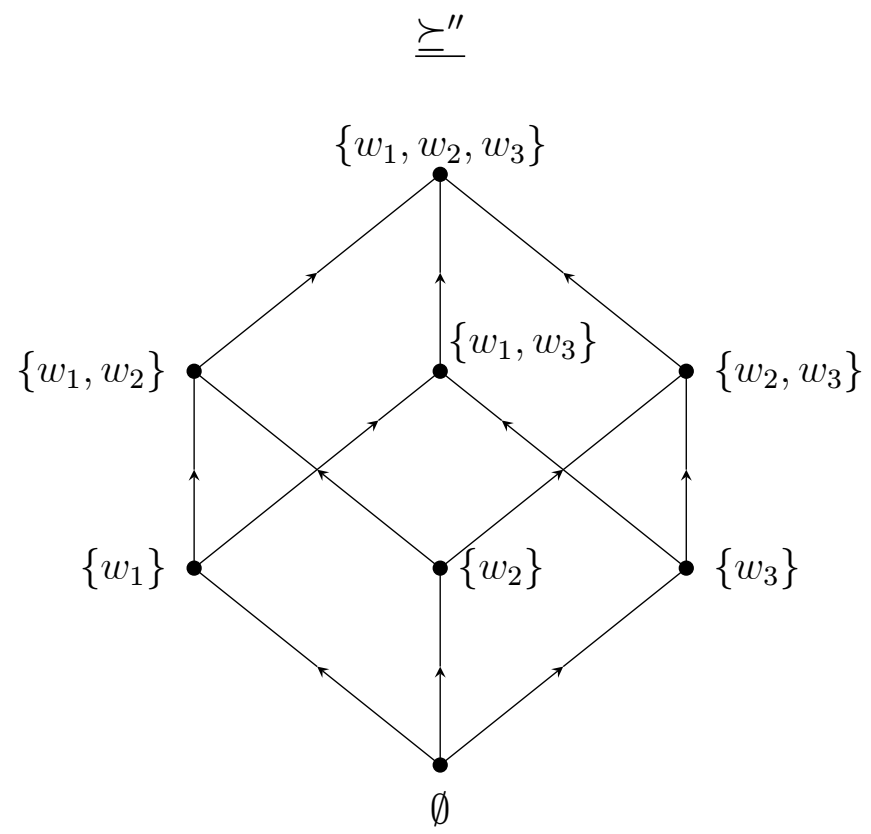


is individually rational, ordered, and closed.

\section{Preliminary Results and Strong Extensions}

Our next result says that the semilattice of the choice of the union $L_{P_{f}}=\left(A_{P_{f}}, \succeq_{P_{f}}, \vee_{P_{f}}\right)$ obtained from a substitutable preference relation $P_{f}$ by conditions (2) and (3) is individually rational, ordered, and closed.

Proposition 2 Let $P_{f}$ be a substitutable preference relation on $2^{W}$. Then, $L_{P_{f}}=\left(A_{P_{f}}, \succeq_{P_{f}}\right.$ ,$\left.\vee_{P_{f}}\right)$ is individually rational, ordered, and closed.

Proof The semilattice $L_{P_{f}}=\left(A_{P_{f}}, \succeq_{P_{f}}, \vee_{P_{f}}\right)$ is individual rational trivially.

To show that it is ordered, let $S, S^{\prime} \in A_{P_{f}}$. First, as we have already argued when showing that $L_{P_{f}}$ is a semilattice, condition (4) holds; i.e.,

$$
l u b_{\succeq_{P_{f}}}\left\{S, S^{\prime}\right\}=C h\left(S \cup S^{\prime}, P_{f}\right) .
$$

We claim that

$$
C h\left(S \cup S^{\prime}, P_{f}\right)=l u b_{\succeq_{P_{f}}}\left\{T \in A_{P_{f}} \mid T \subseteq S \cup S^{\prime}\right\} .
$$

To see it, note that $C h\left(S \cup S^{\prime}, P_{f}\right) \succeq_{P_{f}} T$ for all $T \in A_{P_{f}}$ such that $T \subseteq S \cup S^{\prime}$. By the definition of $A_{P_{f}}, C h\left(S \cup S^{\prime}, P_{f}\right) \in\left\{T \in A_{P_{f}} \mid T \subseteq S \cup S^{\prime}\right\}$. Hence, (7) follows.

To show that $L_{P_{f}}$ is closed suppose that $S \in A_{P_{f}}$ and $S^{\prime} \subseteq S$. By definition of $A_{P_{f}}$, $S=C h\left(S, P_{f}\right)$. Since $P_{f}$ is substitutable, for any $w \in S, C h\left(S \backslash\{w\}, P_{f}\right)=S \backslash\{w\}$. Iterating this property, if necessary, we obtain that $S^{\prime}=C h\left(S^{\prime}, P_{f}\right)$. Hence, $S^{\prime} \in A_{P_{f}}$. Thus, $L_{P_{f}}$ is closed.

Now, we will change our point of view. Previously, starting from a substitutable preference relation $P_{f}$ we eliminated some orderings in $P_{f}$ and constructed, using conditions (2) and (3), an individually rational, ordered, and closed semilattice $\left(A_{P_{f}}, \succeq_{P_{f}}, \vee_{P_{f}}\right)$. Consider now a semilattice $L=(A, \succeq, \vee)$. Since $\succeq$ may leave some pairs $S, S^{\prime} \in A$ unordered, we may complete $\succeq$ to make it a preference relation. A particular way (there are many) of completing $\succeq$ will be called a strong extension of the semilattice.

Definition 8 Let $f \in F$ be given. A preference relation $P_{f}$ on $2^{W}$ is a strong extension of the semilattice $L=(A, \succeq, \vee)$ if the following two properties hold: 
(E.1) For all $S, S^{\prime} \in A, S^{\prime} \succ S$ implies $S^{\prime} P_{f} S$.

(E.2) If $S \notin A$, then there exists $S^{\prime} \in A$ such that $S^{\prime} \subseteq S$ and $S^{\prime} P_{f} S$.

Definition 8 can be interpreted as a set of instructions on how to extend a partial order on $A$ to a complete preference relation on $2^{W}$. First, it has to keep all already existing orderings (this corresponds to the standard notion of an extension of a partial order as used by Szpilrajn (1930)). Second, if a set is not an element of $A$, then we have freedom but the set has to be worse than one of its subsets in $A$ (for instance, the empty set). Finally, pairs of sets in $A$ that are not ordered by $\succeq$ can be freely ordered. The fact $\emptyset \in A$ guarantees that, given a semilattice, we can always find a strong extension. Again, conditions (E.1) and (E.2) do not uniquely identify a preference relation as a strong extension of a semilattice. The preferences $P_{f_{2}}$ and $P_{f_{2}}^{\prime}$ in Example 1 are strong extensions of $\succeq_{P_{f_{2}}}$ while $P_{f_{2}}^{\prime \prime}$ is not. Our main goal below will be to identify for each firm equivalence classes of substitutable preference relations with the property that all members in the same class are strong extensions of the same semilattice.

The next two results are instrumental and they will be useful later on.

Lemma 1 Let $P_{f}$ be a strong extension of the semilattice $L=(A, \succeq, \vee)$. Then, for all $S \in 2^{W}, C h\left(S, P_{f}\right) \in A$.

Proof Assume $S \in 2^{W}$ is such that $C h\left(S, P_{f}\right) \notin A$. Since $P_{f}$ is a strong extension of $L$, there exists $S^{\prime} \in A, S^{\prime} \varsubsetneqq C h\left(S, P_{f}\right)$ such that

$$
S^{\prime} P_{f} C h\left(S, P_{f}\right)
$$

But $S^{\prime} \subseteq S$ contradicts the definition of choice set, because $C h\left(S, P_{f}\right)$ is the most preferred subset of $S$ according to $P_{f}$.

Lemma 2 Assume $L=(A, \succeq, \vee)$ is an individually rational, ordered, and closed semilattice. Then, for all $S, S^{\prime} \in A$ and all $w \in S \vee S^{\prime}, w \in S \vee\{w\}$.

Proof Assume otherwise; that is, there exist $S, S^{\prime} \in A$ and $w \in S \vee S^{\prime}$ such that $w \notin$ $S \vee\{w\}$. Observe that, by closedness, $\{w\} \in A$. We will show that

$$
S=S \vee\{w\} .
$$


By definition of $\vee$ and orderedness, $S \vee\{w\}=l u b_{\succeq}\{S,\{w\}\} \subseteq S \cup\{w\}$. Since, by hypothesis, $w \notin S \vee\{w\}, S \vee\{w\} \subseteq S$. On the other hand, by individual rationality, $S \succeq S \vee\{w\}$. By the definition of $\vee, S \vee\{w\} \succeq S$. Hence, (8) holds.

Define $R=\left(S \vee S^{\prime}\right) \backslash\{w\}$. By closedness, $R \in A$. By (8), $R \vee S=R \vee(S \vee\{w\})$. By orderedness, $R \vee(S \vee\{w\}) \subseteq R \cup(S \vee\{w\})$. Hence, and since $w \notin R$ and $w \notin S \vee\{w\}$, $w \notin R \vee S \subset S \cup S^{\prime}$ and $w \in S \cup S^{\prime}$. Thus,

$$
S \vee S^{\prime} \succ R \vee S
$$

Therefore,

$$
\begin{array}{rlr}
R \vee S \vee\{w\} & =R \vee S \quad \text { by }(8) \\
& \prec S \vee S^{\prime} \quad \text { by }(9) .
\end{array}
$$

Now, we claim that

$$
R \vee\{w\}=S \vee S^{\prime}
$$

First, by orderedness, $R \vee\{w\} \subseteq R \cup\{w\}$, and by definition of $R, R \cup\{w\}=S \vee S^{\prime}$. Hence, by orderedness, $R \vee\{w\} \subseteq S \vee S^{\prime}$. By closedness, $S \vee S^{\prime} \succeq R \vee\{w\}$. Second, by orderedness, $R \vee\{w\}=l u b_{\succeq}\{T \in A \mid T \subseteq R \cup\{w\}\}$. By definition of $R, R \vee\{w\}=l u b_{\succeq}\{T \in A \mid T \subseteq$ $\left.S \vee S^{\prime}\right\}$. Thus, $R \vee\{w\} \succeq S \vee S^{\prime}$. The two orderings imply that (10) holds. Therefore,

$$
\begin{aligned}
R \vee S \vee\{w\} & =R \vee\{w\} \vee S & & \text { because } \vee \text { is commutative } \\
& =\left(S \vee S^{\prime}\right) \vee S & & \text { by }(10) \\
& =S \vee S^{\prime} & & \text { because } \vee \text { is commutative and idempotent, }
\end{aligned}
$$

which contradicts that $R \vee S \vee\{w\} \prec S \vee S^{\prime}$

In Proposition 3 we say that all strong extensions of individually rational, ordered, and closed semilattices are substitutable.

Proposition 3 Let $L=(A, \succeq, \vee)$ be an individually rational, ordered, and closed semilattice and assume $P_{f}$ is a strong extension of $L$. Then, $P_{f}$ is substitutable.

Proof Assume $P_{f}$ is not substitutable. Then, there exist $S$ and $w, w^{\prime} \in S\left(w \neq w^{\prime}\right)$ such that $w \in C h\left(S, P_{f}\right) \equiv \bar{S}$ and

$$
w \notin C h\left(S \backslash\left\{w^{\prime}\right\}, P_{f}\right) \equiv \bar{S}^{\prime} .
$$


By Lemma $1, \bar{S}, \bar{S}^{\prime} \in A$. We will prove that $\bar{S}=\bar{S} \vee \bar{S}^{\prime}$. Assume otherwise; then, $\left(\bar{S} \vee \bar{S}^{\prime}\right) \succ$ $\bar{S}$. Since $P_{f}$ is a strong extension of $L$,

$$
\left(\bar{S} \vee \bar{S}^{\prime}\right) P_{f} \bar{S}
$$

but

$$
\bar{S} \vee \bar{S}^{\prime} \subseteq \bar{S} \cup \bar{S}^{\prime} \subseteq S
$$

The first inclusion holds because $L$ is ordered and the second one by the definition of the choice set. Conditions (12) and (13) contradict that $C h\left(S, P_{f}\right)=\bar{S}$. Hence, $w \in \bar{S} \vee \bar{S}^{\prime}$. Because $L$ is closed, $\{w\} \in A$. By Lemma 2, $w \in \bar{S}^{\prime} \vee\{w\}$. Hence, and since $w \notin \bar{S}^{\prime}$, $\bar{S}^{\prime} \vee\{w\} \succ \bar{S}^{\prime}$. Thus, because $P_{f}$ is a strong extension of $L$,

$$
\left(\bar{S}^{\prime} \vee\{w\}\right) P_{f} \bar{S}^{\prime}
$$

By orderedness, $\bar{S}^{\prime} \vee\{w\} \subseteq \bar{S}^{\prime} \cup\{w\}$. Moreover, $S \backslash\left\{w^{\prime}\right\} \supseteq \bar{S}^{\prime} \cup\{w\}$ since $\bar{S}^{\prime} \subseteq S \backslash\left\{w^{\prime}\right\}$ and $w \in S \backslash\left\{w^{\prime}\right\}$. Then, (14) contradicts that $C h\left(S \backslash\left\{w^{\prime}\right\}, P_{f}\right)=\bar{S}^{\prime}$.

The non closed semilattice $L^{\prime}=\left(A, \succeq^{\prime}, \vee^{\prime}\right)$ of Example 3 shows that there are strong extensions of individually rational and ordered semilattices that are not substitutable. For instance, the strong extension

$$
\begin{gathered}
\frac{P_{f}^{\prime}}{\left\{w_{1}, w_{2}, w_{3}\right\}} \\
\left\{w_{1}, w_{2}\right\} \\
\left\{w_{1}, w_{3}\right\} \\
\left\{w_{2}, w_{3}\right\}
\end{gathered}
$$

$\emptyset$

is not substitutable since $w_{1} \in C h\left(\left\{w_{1}, w_{2}\right\}, P_{f}\right)$ while $w_{1} \notin C h\left(\left\{w_{1}\right\}, P_{f}\right)=\emptyset$.

In Proposition 4 we state that the following consistency property holds. Suppose we start with an individually rational, ordered, and closed semilattice, and strongly extend it. Then, using conditions (2) and (3), we can construct from this strong extension the semilattice of the choice of the union. Then, this semilattice coincides with the semilattice that we started with. Formally,

Proposition 4 Let $P_{f}$ be a strong extension of an individually rational, ordered, and closed semilattice $L=(A, \succeq, \vee)$. Then, $L=\left(A_{P_{f}}, \succeq_{P_{f}}, \vee_{P_{f}}\right)$. 
Proof First, we will show that $A=A_{P_{f}}$. By Lemma 1, $A_{P_{f}} \subseteq A$. Now, we show that if $S \in A$ then $S \in A_{P_{f}}$. Assume $S \notin A_{P_{f}}$; i.e., $S \neq C h\left(S, P_{f}\right)$. By $\operatorname{Lemma} 1, C h\left(S, P_{f}\right) \in$ $A$. Since $L$ is individually rational and $S \supseteq C h\left(S, P_{f}\right), S \succeq C h\left(S, P_{f}\right)$. Since $P_{f}$ is a strong extension of $L, S R_{f} C h\left(S, P_{f}\right)$. But, $S \neq C h\left(S, P_{f}\right)$ implies $S P_{f} C h\left(S, P_{f}\right)$, which contradicts the definition of the choice set. Thus, $A \subseteq A_{P_{f}}$.

Second, we will show that if $S, S^{\prime} \in A$ then $S \vee S^{\prime}=C h\left(S \cup S^{\prime}, P_{f}\right)$. By Lemma 1, $C h\left(S \cup S^{\prime}, P_{f}\right) \in A$. Since $C h\left(S \cup S^{\prime}, P_{f}\right) \subseteq S \cup S^{\prime}$ and $S \vee S^{\prime}=l u b_{\succeq}\left\{T \in A \mid T \subseteq S \cup S^{\prime}\right\}$, by orderedness, $\left(S \vee S^{\prime}\right) \succeq C h\left(S \cup S^{\prime}, P_{f}\right)$. Since $P_{f}$ is a strong extension of $L$,

$$
\left(S \vee S^{\prime}\right) R_{f} C h\left(S \cup S^{\prime}, P_{f}\right)
$$

Since $L$ is ordered, $\left(S \vee S^{\prime}\right) \subseteq\left(S \cup S^{\prime}\right)$. Thus,

$$
C h\left(S \cup S^{\prime}, P_{f}\right) R_{f}\left(S \vee S^{\prime}\right)
$$

Conditions (15) and (16) imply that

$$
S \vee S^{\prime}=C h\left(S \cup S^{\prime}, P_{f}\right)
$$

By the definition of $\vee_{P_{f}}, S \vee_{P_{f}} S^{\prime}=l u b_{\succeq_{P_{f}}}\left\{S, S^{\prime}\right\}$. By condition (4), $S \vee_{P_{f}} S^{\prime}=C h(S \cup$ $\left.S^{\prime}, P_{f}\right)$. By (17), $S \vee S^{\prime}=S \vee_{P_{f}} S^{\prime}$.

\section{The Invariance Result}

We are now ready to state and prove our main result of the paper.

Theorem 1 Let $P_{f}$ and $P_{f}^{\prime}$ be two substitutable preference relations of $f \in F$. Then,

$$
L_{P_{f}}=L_{P_{f}^{\prime}} \text { if and only if } S\left(P_{f}, P_{-f}\right)=S\left(P_{f}^{\prime}, P_{-f}\right) \text { for all substitutable } P_{-f} \text {. }
$$

Proof $(\Rightarrow)$ Let $P_{f}$ and $P_{f}^{\prime}$ be two substitutable preference relations such that $L_{P_{f}}=L_{P_{f}^{\prime}}$. Assume that $P_{-f}$ and $\mu$ are such that $\mu \in S\left(P_{f}, P_{-f}\right)$. Then, $\mu \in I R\left(P_{f}, P_{-f}\right)$. Hence, for all $\hat{f} \in F$ and all $w \in W$,

$$
\mu(\hat{f})=C h\left(\mu(\hat{f}), P_{\hat{f}}\right) \text { and } \mu(w) R_{w} \emptyset .
$$


By Lemma $1, \mu(f) \in A_{P_{f}}$. Then, $A_{P_{f}}=A_{P_{f}^{\prime}}$ implies $\mu(f)=C h\left(\mu(f), P_{f}^{\prime}\right)$. Condition (18) implies that $\mu \in I R\left(P_{f}^{\prime}, P_{-f}\right)$. Assume $\mu \notin S\left(P_{f}^{\prime}, P_{-f}\right)$. Then, there exist $\hat{f}$ and $w$ such that $(\hat{f}, w)$ blocks $\mu$ at $P^{\prime}=\left(P_{f}^{\prime}, P_{-f}\right)$; i.e., $w \notin \mu(\hat{f})$,

$$
\hat{f} P_{w}^{\prime} \mu(w)
$$

and

$$
w \in C h\left(\mu(\hat{f}) \cup\{w\}, P_{\hat{f}}^{\prime}\right) .
$$

Since $P_{w}^{\prime}=P_{w},(19)$ is equivalent to

$$
\hat{f} P_{w} \mu(w) .
$$

If $\hat{f} \neq f$, then $(\hat{f}, w)$ blocks $\mu$ at $\left(P_{f}, P_{-f}\right)$, which contradicts that $\mu \in S\left(P_{f}, P_{-f}\right)$. Hence, $\hat{f}=f$. By Lemma $1, C h\left(\mu(f) \cup\{w\}, P_{f}^{\prime}\right) \in A_{P_{f}^{\prime}}$, and since, by Proposition 2 , the semilattice $L_{P_{f}^{\prime}}$ is closed, $\{w\} \in A_{P_{f}^{\prime}}$. Condition $(20)$ and $w \notin \mu(f)$ imply

$$
\left(\mu(f) \vee_{P_{f}^{\prime}}\{w\}\right) \succ_{P_{f}^{\prime}} \mu(f) .
$$

By $f P_{w} \mu(w)$ and $\mu \in S\left(P_{f}, P_{-f}\right),(20)$ implies

$$
\mu(f)=\mu(f) \vee_{P_{f}}\{w\} .
$$

This contradicts $(22)$ because $L_{P_{f}}=L_{P_{f}^{\prime}}$. Then, $S\left(P_{f}, P_{-f}\right) \subseteq S\left(P_{f}^{\prime}, P_{-f}\right)$. Hence, $S\left(P_{f}, P_{-f}\right)=$ $S\left(P_{f}^{\prime}, P_{-f}\right)$.

$(\Leftarrow)$ Let $P_{f}$ and $P_{f}^{\prime}$ be two substitutable preference relations and assume $S\left(P_{f}, P_{-f}\right)=$ $S\left(P_{f}^{\prime}, P_{-f}\right)$ for all substitutable $P_{-f}$. To show that $L_{P_{f}}=L_{P_{f}^{\prime}}$, we first show $S \in A_{P_{f}}$ if and only if $S \in A_{P_{f}^{\prime}}$. Consider the following preference profile $P_{-f}$ : for all $w \in S$, all $w^{\prime} \notin S$, and all $\hat{f} \neq f$,

$$
\frac{P_{w}}{f} \quad \frac{P_{w^{\prime}}}{\emptyset} \quad \frac{P_{\hat{f}}}{\emptyset}
$$

$\emptyset$.

The unique stable matching at $\left(P_{f}, P_{-f}\right)$ is $\mu$, where $\mu(f)=S$ and $\mu(\hat{f})=\emptyset$ for all $\hat{f} \neq f$ (obviously, $\mu\left(w^{\prime}\right)=\emptyset$ for all $w^{\prime} \notin S$ ). By hypothesis, $S\left(P_{f}, P_{-f}\right)=S\left(P_{f}^{\prime}, P_{-f}\right)$. Hence, $\mu$ is individually rational at $\left(P_{f}^{\prime}, P_{-f}\right)$. Then, $S=\mu(f)=C h\left(\mu(f), P_{f}^{\prime}\right)=C h\left(S, P_{f}^{\prime}\right)$. By Lemma $1, S \in A_{P_{f}}$. 
To prove that $\succeq_{P_{f}}=\succeq_{P_{f}^{\prime}}$, we will first show that for any $S_{1}, S_{2} \in A_{P_{f}}$,

$$
S_{1}=S_{1} \vee_{P_{f}} S_{2} \text { if and only if } S_{1}=S_{1} \vee_{P_{f}^{\prime}} S_{2}
$$

Observe that if $F=\{f\}$ then the result follows trivially by the definitions of $\succeq_{P_{f}}$ and $\succeq_{P_{f}^{\prime}}$. Thus, assume $|F| \geq 2$. Consider any preference profile $P_{-f}$ where, for a firm $f^{\prime} \neq f$, for all $w \in S_{1} \backslash S_{2}$, all $w^{\prime} \in S_{1} \cap S_{2}$, all $w^{\prime \prime} \in S_{2} \backslash S_{1}$, all $w^{\prime \prime \prime} \notin S_{1} \cup S_{2}$, and all $\hat{f} \neq f, f^{\prime}$,

\begin{tabular}{|c|c|c|c|c|}
\hline$\underline{P_{w}}$ & $\underline{P_{w^{\prime}}}$ & $\underline{P_{w^{\prime \prime}}}$ & $\underline{P_{w^{\prime \prime \prime}}}$ & $\underline{P_{f^{\prime}}}$ \\
\hline$f^{\prime}$ & $f$ & $f$ & $\emptyset$ & $S_{2} \backslash S_{1}$ \\
\hline$f$ & $\emptyset$ & $f^{\prime}$ & & $\cdots$ \\
\hline$\emptyset$ & & $\emptyset$ & & $S_{1} \backslash S_{2}$ \\
\hline
\end{tabular}

Observe that $P_{f^{\prime}}$ is not fully described and it may be completed by keeping it substitutable. By $S_{1}=S_{1} \vee_{P_{f}} S_{2}, C h\left(S_{1}, P_{f}\right)=S_{1}$. We claim that for all $T \supseteq S_{1}, C h\left(T, P_{f}\right) \cap\left(S_{2} \backslash S_{1}\right)=$ $\emptyset$. Assume $w \in C h\left(T, P_{f}\right) \cap\left(S_{2} \backslash S_{1}\right) \neq \emptyset$ for some $T \supseteq S_{1}$. By substitutability of $P_{f}$, $w \in C h\left(T, P_{f}\right)$ implies that $w \in C h\left(S_{1}, P_{f}\right)$. Since $w \in S_{2} \backslash S_{1}, w \notin S_{1}$, contradicting that $C h\left(S_{1} \cup S_{2}, P_{f}\right)=S_{1}$, which holds because $S_{1}=S_{1} \vee_{P_{f}} S_{2}$. Thus, the firms-optimal stable matching $\mu_{F} \in S\left(P_{f}, P_{-f}\right)$ is $\mu_{F}(f)=S_{1}, \mu_{F}\left(f^{\prime}\right)=S_{2} \backslash S_{1}$, and $\mu_{F}(\hat{f})=\emptyset$ for all $\hat{f} \neq f, f^{\prime}$. To see that, consider the deferred-acceptance algorithm in which firms propose. Observe first that $f^{\prime}$ proposes in the first step of the algorithm to $S_{2} \backslash S_{1}$, and all these offers are accepted. Moreover, at any step of the algorithm in which $f$ proposes, it proposes to $C h\left(W^{\prime}, P_{f}\right)$ for some set of workers $W^{\prime}$, where $W^{\prime} \supseteq S_{1}$; this is because all workers in $S_{1}$ only receive offers from $f$ and all workers in $S_{1} \backslash S_{2}$ do not receive offers from $f^{\prime}$ since $f^{\prime}$ does not make any offer to the workers in $S_{1}$, and none of the other firms (if any) makes any offer. By hypothesis, $S\left(P_{f}, P_{-f}\right)=S\left(P_{f}^{\prime}, P_{-f}\right)$. Hence, $\mu_{F} \in S\left(P_{f}^{\prime}, P_{-f}\right)$. Moreover, $S_{1}=\mu_{F}(f)=C h\left(\mu_{F}(f), P_{f}^{\prime}\right)$. Assume that $S_{1} \neq S_{1} \vee_{P_{f}^{\prime}} S_{2}$. Then, there exists $w^{\prime \prime} \in S_{2} \backslash S_{1}$ such that $w^{\prime \prime} \in S_{1} \vee_{P_{f}^{\prime}} S_{2}$. By Lemma 2, $w^{\prime \prime} \in S_{1} \vee_{P_{f}^{\prime}}\left\{w^{\prime \prime}\right\}$. Then, $S_{1} \vee_{P_{f}^{\prime}}\left\{w^{\prime \prime}\right\} \succ_{P_{f}^{\prime}} S_{1}$. Because $L_{P_{f}^{\prime}}$ is closed, $w^{\prime \prime} \in A_{P_{f}^{\prime}}$. By definition of $L_{P_{f}^{\prime}}, S_{1} \vee_{P_{f}^{\prime}}\left\{w^{\prime \prime}\right\} \succ_{P_{f}^{\prime}} S_{1}$ implies $\left(S_{1} \vee_{P_{f}^{\prime}}\left\{w^{\prime \prime}\right\}\right) P_{f}^{\prime} S_{1}$. Then, $w^{\prime \prime} \in C h\left(\mu_{F}(f) \cup\left\{w^{\prime \prime}\right\}, P_{f}^{\prime}\right)$. But $f P_{w^{\prime \prime}} f^{\prime}$, because $w^{\prime \prime} \in S_{2} \backslash S_{1}$. Thus, $\left(f, w^{\prime \prime}\right)$ blocks $\mu_{F}$ at $\left(P_{f}^{\prime}, P_{-f}\right)$, which contradicts that $\mu_{F} \in S\left(P_{f}^{\prime}, P_{-f}\right)$. Hence, 
$S_{1}=S_{1} \vee_{P_{f}^{\prime}} S_{2}$. Finally,

$$
\begin{aligned}
S_{1} \succeq_{P_{f}} S_{2} & \Longleftrightarrow S_{1}=S_{1} \vee_{P_{f}} S_{2} & & \text { by condition (3) and orderedness of } L_{P_{f}} \\
& \Longleftrightarrow S_{1}=S_{1} \vee_{P_{f}^{\prime}} S_{2} & & \text { by }(24) \\
& \Longleftrightarrow S_{1} \succeq_{P_{f}^{\prime}} S_{2} & & \text { by condition (3) and orderedness of } L_{P_{f}^{\prime}} .
\end{aligned}
$$

Thus, $L_{P_{f}}=L_{P_{f}^{\prime}}$ because $A_{P_{f}}=A_{P_{f}^{\prime}}$ and $\succeq_{P_{f}}=\succeq_{P_{f}^{\prime}}$ implies $\vee_{P_{f}}=\vee_{P_{f}^{\prime}}$.

Our result on the invariance of the set of stable matchings requires substitutability. In Example 4 we show that there are two non-substitutable preference relations $P_{f}$ and $P_{f}^{\prime}$ with the property that $S\left(P_{f}, P_{-f}\right)=S\left(P_{f}^{\prime}, P_{-f}\right)$ for all $P_{-f}$ but $L_{P_{f}} \neq L_{P_{f}^{\prime}}$.

Example 4 Let $W=\left\{w_{1}, w_{2}, w_{3}, w_{4}\right\}$ be the set of workers and let $f \in F$. Consider the two preference relations

$$
\begin{aligned}
& \frac{P_{f}}{\left\{w_{1}, w_{2}\right\}} \quad \frac{P_{f}^{\prime}}{\left\{w_{3}, w_{4}\right\}} \\
& \left\{w_{3}, w_{4}\right\} \quad\left\{w_{1}, w_{2}\right\}
\end{aligned}
$$

$\emptyset \quad \emptyset$.

Observe that neither $P_{f}$ nor $P_{f}^{\prime}$ are substitutable. Moreover, although $A_{P_{f}}=A_{P_{f}^{\prime}}=$ $\left\{\left\{w_{1}, w_{2}\right\},\left\{w_{3}, w_{4}\right\}, \emptyset\right\} \subset 2^{W}$ the two semilattices of the choice of the union obtained by conditions (2) and (3) from $P_{f}$ and $P_{f}^{\prime}$ are different and, respectively, equal to

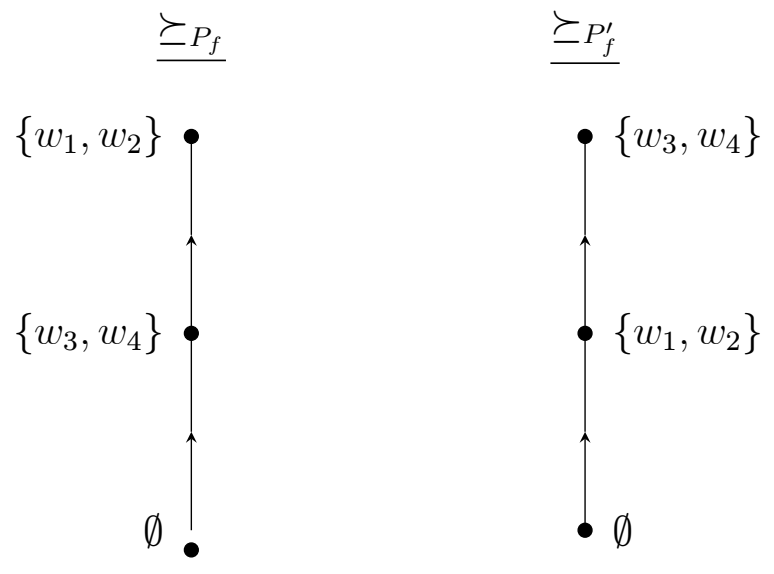

Hence, $L_{P_{f}} \neq L_{P_{f}^{\prime}}$. Let $P_{-f}$ be arbitrary. Assume $\mu \in S\left(P_{f}, P_{-f}\right)$. We will show that $\mu \in S\left(P_{f}^{\prime}, P_{-f}\right)$. First, assume $\mu \notin S\left(P_{f}^{\prime}, P_{-f}\right)$ and let $P^{\prime}=\left(P_{f}^{\prime}, P_{-f}\right)$. Since $I R\left(P_{f}, P_{-f}\right)=$ 
$I R\left(P_{f}^{\prime}, P_{-f}\right)$, there exist $\hat{f} \in F$ and $w \in W$ such that $(\hat{f}, w)$ blocks $\mu$ at $\left(P_{f}^{\prime}, P_{-f}\right) ;$ i.e.,

$$
\hat{f} P_{w}^{\prime} \mu(w)
$$

and

$$
w \in C h\left(\mu(\hat{f}) \cup\{w\}, P_{\hat{f}}^{\prime}\right) .
$$

Since $P_{w}=P_{w}^{\prime},(25)$ implies $\hat{f} P_{w} \mu(w)$. If $\hat{f} \neq f$, then $(\hat{f}, w)$ blocks $\mu$ at $\left(P_{f}, P_{-f}\right)$ and this contradicts that $\mu \in S\left(P_{f}, P_{-f}\right)$. Hence, $\hat{f}=f$. We consider the following three cases, depending on the set $\mu(f)$ :

Case 1: $\mu(f)=\left\{w_{1}, w_{2}\right\}$. Then, for all $w \notin \mu(f), \mu(f)=C h\left(\mu(f) \cup\{w\}, P_{f}^{\prime}\right)$. Thus, for all $w \notin \mu(f),(f, w)$ does not block $\mu$ at $\left(P_{f}^{\prime}, P_{-f}\right)$.

Case 2: $\mu(f)=\left\{w_{3}, w_{4}\right\}$. Then, for all $w \notin \mu(f), \mu(f)=C h\left(\mu(f) \cup\{w\}, P_{f}^{\prime}\right)$. Thus, for all $w \notin \mu(f),(f, w)$ does not block $\mu$ at $\left(P_{f}^{\prime}, P_{-f}\right)$.

Case 3: $\mu(f)=\emptyset$. Then, for all $w \notin \mu(f)$, we have that $\mu(f)=C h\left(\mu(f) \cup\{w\}, P_{f}^{\prime}\right)$. Thus, for all $w \notin \mu(f),(f, w)$ does not block $\mu$ at $\left(P_{f}^{\prime}, P_{-f}\right)$.

These three cases show that $S\left(P_{f}, P_{-f}\right) \subseteq S\left(P_{f}^{\prime}, P_{-f}\right)$. Using a similar argument we can show that $S\left(P_{f}^{\prime}, P_{-f}\right) \subseteq S\left(P_{f}, P_{-f}\right)$. Then, for all $P_{-f}, S\left(P_{f}, P_{-f}\right)=S\left(P_{f}^{\prime}, P_{-f}\right)$, and $L_{P_{f}} \neq L_{P_{f}^{\prime}}$.

\section{Concluding Remark}

The main implication of Theorem 1 is the following. Let $P=\left(P_{f_{1}}, \ldots, P_{f_{n}} ; P_{w_{1}}, \ldots, P_{w_{m}}\right)$ be a substitutable profile of preference relations of agents. For each $f_{i} \in F, i=1, . ., n, P_{f_{i}}$ is a complete order on a set of cardinality $2^{|W|}$. Identify, using (2), the subfamily of subsets of workers $A_{P_{f_{i}}}$ that are the choice of themselves. Construct, using (3), the partial order $\succeq_{P_{f_{i}}}$ on $A_{P_{f_{i}}}$. All information needed to compute the set of stable matchings at preference profile $P$ is embedded in the profile $\succeq=\left(\succeq_{P_{f_{1}}}, \ldots, \succeq_{P_{f_{n}}} ; P_{w_{1}}, \ldots, P_{w_{m}}\right)$. Moreover, any preference profile $P^{\prime}=\left(P_{f_{1}}^{\prime}, \ldots, P_{f_{n}}^{\prime} ; P_{w_{1}}, \ldots, P_{w_{m}}\right)$ such that $\succeq_{P_{f_{i}}^{\prime}}=\succeq_{P_{f_{i}}}$ for all $i=1, \ldots, n$ has the property that $S(P)=S\left(P^{\prime}\right)$. Hence, we can partition the set of substitutable preference profiles into equivalence classes in such a way that all preference profiles in the same class have the same set of stable matchings. A profile $\succeq=\left(\succeq_{f_{1}}, \ldots, \succeq_{f_{n}} ; P_{w_{1}}, \ldots, P_{w_{m}}\right)$ 
of partial orders may be used as the representative of each class. All substitutable preference profiles in which preference relations of firms are strong extensions of their corresponding partial order belong to the same equivalence class (see the figure below). Thus, any centralized mechanism that proposes a stable matching for each preference profile $P=\left(P_{f_{1}}, \ldots, P_{f_{n}} ; P_{w_{1}}, \ldots, P_{w_{m}}\right)$ can use as input, instead, the corresponding profile of partial orders $\succeq=\left(\succeq_{P_{f_{1}}}, \ldots, \succeq_{P_{f_{n}}} ; P_{w_{1}}, \ldots, P_{w_{m}}\right)$.

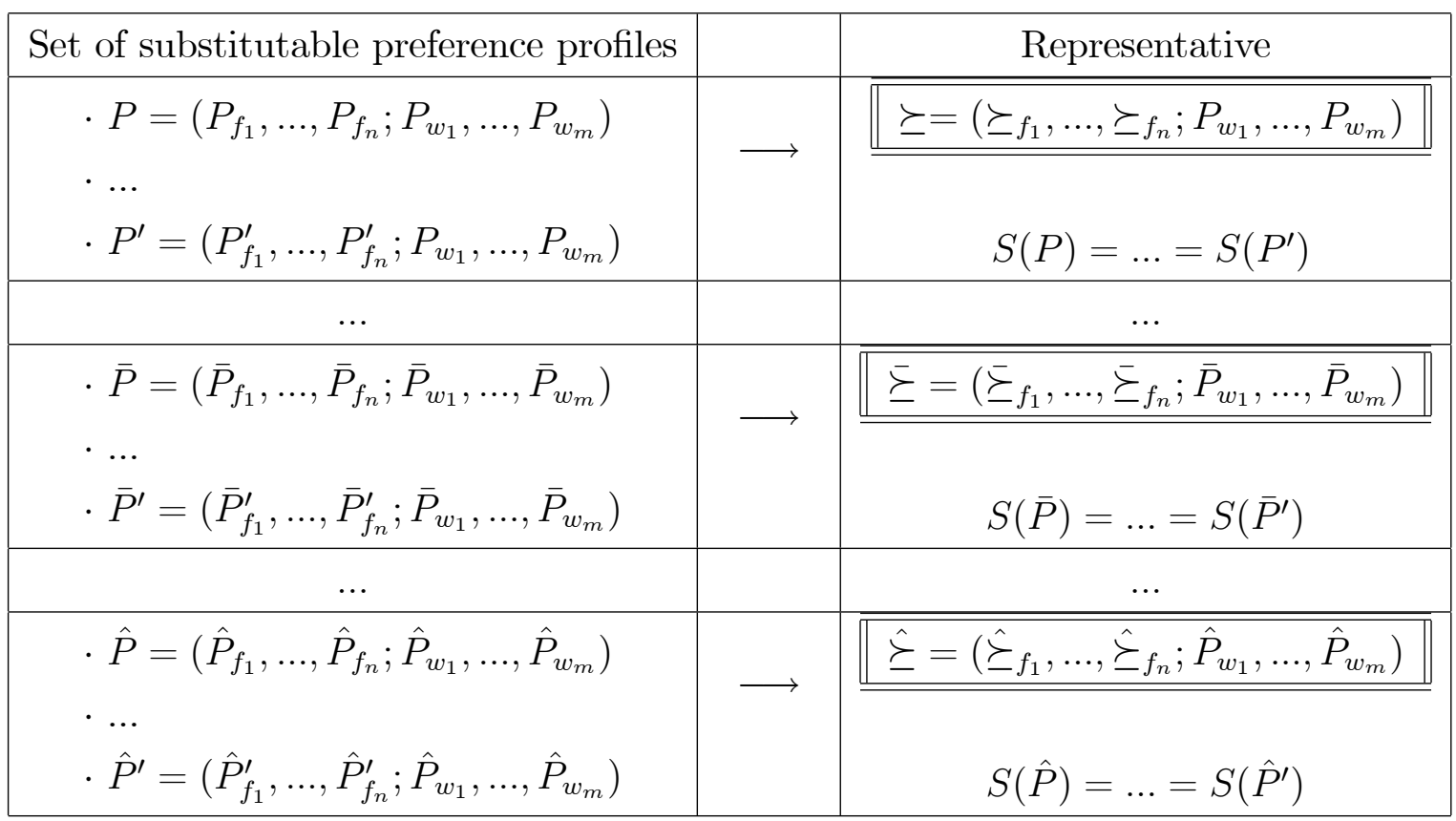

\section{References}

[1] Abdulkadiroğlu, A., Pathak, P., and Roth, A. "The New York City High School Match," American Economic Review, Papers and Proceedings 95, 364-367 (2005).

[2] Abdulkadiroğlu, A., Pathak, P., Roth, A., and Sönmez, T. "The Boston Public School Match," American Economic Review, Papers and Proceedings, 95, 368-371 (2005).

[3] Abdulkadiroğlu, A., Pathak, P., Roth, A., and Sönmez, T. "Changing the Boston School Choice Mechanism: Strategy-Proofness as Equal Access," mimeo (2006). 
[4] Abdulkadiroğlu, A. and Sönmez, T. "School Choice: A Mechanism Design Approach," American Economic Review 93, 729-747 (2003).

[5] Alkan, A. "On Preferences over Subsets and the Lattice Structure of Stable Matchings," Review of Economic Design 6, 99-111 (2001).

[6] Birkhoff, G. Lattice Theory. American Mathematical Society, Colloquium Publications 25; third edition (1979).

[7] Blair, C. "The Lattice Structure of the Set of Stable Matchings with Multiple Partners," Mathematics of Operations Research 13, 619-628 (1988).

[8] Echenique, F. and Oviedo, J. "A Theory of Stability in Many-to-many Matching Markets," Theoretical Economics 1, 233-273 (2006).

[9] Fleiner, T. "A Fixed-point Approach to Stable Matchings and Some Applications," Mathematics of Operations Research 28, 103-126 (2003).

[10] Gale, D. and Shapley, L. "College Admissions and the Stability of Marriage," American Mathematical Monthly 69, 9-15 (1962).

[11] Kelso, A. and Crawford, V. "Job Matchings, Coalition Formation, and Gross Substitutes," Econometrica 50, 1483-1504 (1982).

[12] Romero-Medina, A. and Triossi, M. "Ramón y Cajal: Mediation and Meritocracy," mimeo (2005).

[13] Roth, A. "The Evolution of the Labor Market for Medical Interns and Residents: A Case Study in Game Theory," Journal of Political Economy 92, 991-1016 (1984a).

[14] Roth, A. "Stability and Polarization of Interests in Job Matching," Econometrica 52, 47-57 (1984b).

[15] Roth, A. "Conflict and Coincidence of Interest in Job Matching," Mathematics of Operations Research 10, 379-389 (1985).

[16] Roth, A. "The Economist as Engineer: Game Theory, Experimentation, and Computation as Tools for Design Economics," Econometrica 70, 1341-1378 (2002). 
[17] Roth, A. and Peranson, E. "The Redesign of the Matching Market for American Physicians: Some Engineering Aspects of Economic Design," American Economic Review 89, 748-780 (1999).

[18] Roth, A. and Xing, X. "Jumping the Gun: Imperfections and Institutions Related to the Timing of Market Transactions," American Economic Review 84, 992-1044 (1994).

[19] Szpilrajn, E. "Sur l'extension de l'ordre partiel," Fundamenta Mathematicae 16, 386389 (1930). 